\title{
EACH HOMOGENEOUS NONDEGENERATE CHAINABLE CONTINUUM IS A COSET SPACE
}

\section{TA-SUN WU ${ }^{1}$}

Let $X$ be a compact Hausdorff homogeneous space and $G$ be the full group of homeomorphisms from $X$ to $X$. We denote the isotropy group at $x$ by $G_{x}$. If $G$ is topologized by uniform convergence [1], then it is well known that $G$ is a topological group, and the function $\theta: G / G_{x} \rightarrow X$, defined by $\theta\left(g G_{x}\right)=g(x)$ is one-one and continuous. It is an open problem whether $\theta$ is a homeomorphism, i.e., whether $X$ is a coset space.

In this note we prove that each homogeneous nondegenerate chainable continuum $M$ is such a space.

Ford $[2]^{2}$ has proved that every SLH (strongly locally homogeneous) completely regular Hausdorff space is a coset space. We will prove that $M$ is not an SLH space but still a coset space.

Bing [3] has proved that each homogeneous nondegenerate chainable space is a pseudo-arc. Therefore we only need to prove that the pseudo-arc is a coset space. In his papers $[3 ; 4 ; 5]$, Bing gave a very beautiful treatment of the pseudo-arc. We will follow his definitions and notations, and we will not repeat all his definitions here.

Description of the pseudo-arc $M[4$, p. 730]: Let $P$ and $Q$ be two points of a compact metric space and $D_{1}, D_{2}, \cdots$ a sequence of chains from $P$ to $Q$ such that for each positive integer $i$, (1) $D_{i+1}$ is crooked in $D_{i}$, (2) no link of $D_{i}$ has a diameter of more than $1 / 2^{i}$ and (3) the closure of each link of $D_{i+1}$ is a subset of a link of $D_{i}$. Then $M$ is the common part of $D_{1}^{*}, D_{2}^{*}, \cdots$, where $D_{i}^{*}$ denotes the sum of the links of $D_{i}$.

It is well known that the pseudo-arc is an indecomposable continuum [4].

Definition. A space $X$ is an SLH space if for any $x \in X$, and neighborhood $V$ of $x$, there is a neighborhood $U$ of $x, U \subset V$, such that for any $y \in V$, there is a homeomorphism $g$ of $X$ such that $g(x)=y$ and $g(z)=z$ if $z \notin U$.

Received by the editors September 23, 1960.

1 This paper and another paper under the title $O$ n the reasonable topology of SLH space are offered in partial fulfillment of the requirement for the Master of Science degree at Tulane University under the direction of Professor Paul S. Mostert. I wish to thank Dr. Mostert for his patient and understanding help.

2 The author takes the liberty here of mentioning that Theorem 4.1 in [2] is not correct. Counterexamples and some discussions are given in the author's paper mentioned in Footnote 1. 
Since each composant of the indecomposable continuum $X$ is dense in $X$ and composants are preserved under homeomorphisms, the pseudo-arc $M$ can not be an SLH space.

LEMma. If $M$ is a pseudo-arc, then any open set $U$ meets infinitely many (in fact uncountably many) different composants of $M$.

Proof. Since $M$ has uncountably many composants, and each composant is dense in $M$.

\section{Proposition. $M$ is a coset space. ${ }^{8}$}

Proof. Let $G$ be the full group of homeomorphisms of $M$, which is topologized by uniform convergence. Let $G_{P}$ be the isotropy group at $P$ (cf. description of $M$ ). Since $M$ is a compact metric space, we can define the (unique) uniformity generated by the family of sets $V_{r}=\{(x, y) \mid x, y \in M$ and $\rho(x, y)<r\}$ where $r=1 / 2^{n}, n=1,2, \cdots$, and $\rho(x, y)=$ distance between $x$ and $y$. Let $S(r)$ be the set of all homeomorphisms $g$ of $M$ such that, for all $x \in M, \rho(x, g(x))<r$. By the remark of the first section, all we need to show is that, if $r=1 / 2^{n}$ for some $n$, there is an open set $V$ containing $P$ such that if $y \in V$, there is a homeomorphism $g \in S(r)$ such that $g(P)=y$.

Let $r=1 / 2^{n}$. There is an $i$ such that the diameter of each link of $D_{i}$ is less than $r / 4$. Let $V$ be the first link of $D_{i}$, let $y$ be any point of $V$, and select a point $z$ of the last link of $D_{i}$ such that $z$ and $y$ are in different composants (cf. Lemma).

Using the technique of the proof of Theorem 13 [4], we can find sequences $E_{1 n}, E_{2 n}, \cdots(n=1,2)$ of chains closing down on $M$ such that (1) $E_{j 1}$ is a chain from $P$ to $Q$ and $E_{j 2}$ is a chain from $y$ to $z$, (2) $E_{11}=E_{12}=D_{i}$, (3) no element of $E_{j n}$ has diameter greater than $1 / 2^{j+i}$, (4) $E_{j+1, n}$ is crooked in $E_{j n}$, (5) the closure of each element of $E_{j+1, n}$ is a subset of some element of $E_{j n}$, and (6) $E_{j n}$ is a consolidation of some $D_{k}$.

Now using the techniques of the proof of Theorem 12 [4] followed by those of the proof of Theorem 11 [4] we arrive at a homeomorphism $g$ of $M$ such that, if $x$ is a point of the $j$ th element of $D_{i}, g(x)$ belongs to either the $(j-1)$ th, the $j$ th or $(j+1)$ th element of $D_{i}$ and $g(P)=y$. Clearly, $\rho(x, g(x))<r$, this proves that $g \in S(r)$. Hence, by the remark above, we know that $M$ is a coset space.

\section{REFERENCES}

1. J. L. Kelley, General topology, New York, Van Nostrand, 1955.

3 The author is sincerely grateful for the referee's suggestions and comments on the proof of this proposition. 
2. L. R. Ford, Jr., Homeomorphism groups and coset spaces, Trans. Amer. Math. Soc. vol. 77 (1954) pp. 490-497.

3. R. H. Bing, Each homogeneous nondegenerate chainable continuum is a pseudoarc, Proc. Amer. Math. Soc. vol. 10 (1959) pp. 345-346.

4. - A homogeneous indecomposable plane continuum, Duke Math. J. vol. 15 (1948) pp. 729-742.

5. - Concerning hereditarily indecomposable continua, Pacific J. Math. vol. 1 (1951) pp. 43-51.

TUlANE UNIVERSITY AND

St. Mary's Dominican College

\title{
IMMERSIONS OF ALMOST PARALLELIZABLE MANIFOLDS
}

\author{
MORRIS W. HIRSCH ${ }^{1}$
}

The purpose of this note is to prove the following theorem:

An almost parallelizable $n$-manifold $M$ can be immersed in Euclidean $q$-space $R^{a}$ if $2 q>3 n$.

By immersion $f: M \rightarrow R^{p}$ we mean a continuously differentiable map whose Jacobian matrix has $\operatorname{rank} n=\operatorname{dim} M$ at each point. We denote the differential of an immersion $f$ by $d f$.

A regular homotopy $f_{t}: M \rightarrow R^{n}$ is a homotopy such that each $f_{t}$ is an immersion and $d f_{t}$ is a homotopy of the tangent bundle of $M$ into $R^{n}$. In this case $f_{0}$ and $f_{1}$ have equivalent normal bundles.

We say $M$ is almost parallelizable if the tangent bundle of $M-x$ is trivial, for some $x \in M$.

To prove the theorem, we first observe that if $M$ is not compact, or is bounded, then $M$ is parallelizable, and by [1,6.3], $M$ can be immersed in $R^{n+1} \subset R^{q}$. Hence we assume $M$ is compact and unbounded. Let $B$ be an $n$-ball diffeomorphically embedded in $M$, with bounding $(n-1)$ sphere $S$. Put $M_{0}=M-$ int $B$. By the remark above, there is an immersion $f: M_{0} \rightarrow R^{n+1}$. We consider $f$ as an immersion in $R^{q}$, and we deform $f$ through a regular homotopy near $S$, keeping $f \mid S$ fixed, so that if $X$ is a unit tangent vector to $M$ at point $x \in S$ pointing into $M_{0}$, then $d f(X)$ is the unit vector $e=(0, \cdots, 0,1)$ normal to $R^{q-1}$ in $R^{q}$. We still have $f(S) \subset R^{q-1}$.

Since the immersion $f$ is regularly homotopic to an immersion $M \rightarrow R^{n+1}$, the normal bundle of $f$ is trivial. This enables us to apply a lemma $[2,3.2]$ of M. Kervaire, which implies that the Smale in-

Received by the editors October 10, 1960.

1 Supported by National Science Foundation Contract NSF G-11594. 